Objective The heparan sulphate proteoglycan syndecan-4 (Sdc4) has been associated strongly with osteoarthritis, a disease that mimics key aspects of early cartilage remodelling during endochondral ossification, but its role in embryonic and adult bone formation remains unclear. Therefore, we used $S d \mathrm{c}^{-\%}$ mice to analyse the distribution and functional role of Scd4 in endochondral ossification of mouse embryos and in adult fracture repair, which recapitulates endochondral ossification, but like osteoarthritis, involves an inflammatory component.

Methods $S d c 4$ promoter activity was analysed in $S d c 4 \% / L a c Z$ knock-in animals using $\beta$-galactosidase stainings. E16.5 embyros were used for histological (alcian blue/alizarin red) and immunohistological (PCNA, Col10a1, ADAMTS-4, BC-3, Sdc2) staining and the calcified bone area was quantified using whole mount staining of these embryos. Histological (Masson-Goldner, alcian blue) and immunohistological (Col10a1, Sdc2, PCNA) staining at day 7, 14 and 28 fracture calli were performed. These experiments were repeated with anti-TNF treatment during fracture healing. Callus size and cartilage area were quantified using image J Chondrocytes were isolated from neonatal knee joints and embyronal cartilage. Proliferation was investigated using MTT assay. Gene expression analysis for Sdc-2, Sdc-4 with and without stimulation using TNF $\alpha$ and WNT3a was performed using quantitative RT-PCR.

Results In $S d c 4 \% / L a c Z$ knock-in animals, $S d c 4$ promoter activity was detectable in all stages of chondrocyte differentiation during embryogenesis. Sdc4 deficiency inhibited chondrocyte proliferation both in vivo and in vitro, but this did not lead to a growth phenotype at birth. In contrast to embryogenesis, fracture healing in adult mice was markedly delayed in $S d c 4^{-}$animals and accompanied by increased callus formation. Analysing the discrepancy between the mild embryonic and the severe adult phenotype, we found a compensatory up-regulation of $S d c 2$ in the developing cartilage of $S d c 4^{-\%}$ mice that was absent in adult tissue. Stimulation of chondrocytes with Wnt3a in vitro, led to an increased expression of $S d c 2$, while stimulation with TNF $\alpha$ resulted in an up-regulation of $S d c 4$ but a decreased expression of $S d c 2$. In consequence treatment with a blocking anti-TNF antibody during fracture healing abolished the difference in callus size between wildtype and $s d c 4^{-\%}$ mice.

Conclusions We conclude that Sdc4 is functionally involved in endochondral ossification and that the loss of Sdc4 impairs adult fracture healing due to the inhibition of compensatory mechanisms under inflammatory conditions.

\section{A8.14 THE ANTI-PROLIFERATIVE FUNCTION OF RSK2 IN SYNOVIAL FIBROBLASTS PROTECTS AGAINST TNF- $\alpha$-INDUCED JOINT DESTRUCTION IN INFLAMMATORY ARTHRITIS}

doi:10.1136/annrheumdis-2013-203222.14

\begin{abstract}
1.*Anja Derer, ${ }^{1, *}$ Christina Böhm, 'Bettina Herbort, ${ }^{2}$ Sybille Böhm, ${ }^{3}$ Kirsten Neubert, ${ }^{1}$ Michael Stock, 'Christine Zech, 'Georg Schett, ${ }^{1, *}$ Axel J Hueber ${ }^{14, * *}$ Jean-Pierre David. 'Department of Internal Medicine 3, Rheumatology and Immunology, University Hospital, Erlangen, Germany; ${ }^{2}$ Department of Biology, University of Erlangen-Nuremberg; ${ }^{3}$ Department of Dermatology, Research Modul II, University Hospital, Erlangen; ${ }^{4} / n s t i t u t e$ for Osteology and Biomechanics, University Medical Center Hamburg-Eppendorf, Hamburg, Germany,
\end{abstract}

*Contributed equally

Background/Objectives The pro-inflammatory cytokine Tumor Necrosis Factor alpha (TNF- $\alpha$ ) directly activates the ribosomal S6 kinase RSK2 in vitro. We recently demonstrated the protective effect of RSK2 against TNF-induced bone loss. Interestingly, we found an increased activation of RSK2 in the joints of arthritis patients as well as in the inflamed joints of mice overexpressing the human TNF- $\alpha$ (hTNFtg). These observations prompted us to investigate the function of RSK2 in the development of TNF- $\alpha$ induced inflammatory arthritis.

Materials and Methods hTNFtg mice were crossed with RSK2deficient $\left(R s k 2^{y /-}\right)$ mice. Clinical scoring and histomorphometry of the joints were assessed. We compared the levels of circulating proinflammatory cytokines as well as the cellularity of myeloid lineages in the spleen. The expression of cytokines and mesenchymal markers in the joints was determined via OPCR. Bone marrow transfer of $R s k 2^{y /-}$ and wild-type littermates into hTNFtg mice was performed and clinical scoring as well as histomorphometry of the joints was assessed. Primary fibroblast-like synoviocytes (FLS) from hTNFtg and hTNFtg; Rsk2 $2^{y /}$ mice were isolated to analyse their expression of inflammatory cytokines and metalloproteinases as well as their proliferation and apoptosis in vitro.

Results RSK2 deficiency in $h T N F$ tg mice resulted in an early onset of clinical signs of arthritis as well as a drastic exacerbation of inflammation, increased cartilage destruction and increased local bone destruction. Increased levels of circulating pro-inflammatory cytokines and the increased proportion of all myeloid lineages in the spleen confirmed the enhanced inflammation in the hTNFtg mice lacking RSK2. Increased activation of synovial fibroblasts and macrophages in the joints of hTNFtg; Rsk2\%/ mice was demonstrated by the locally increased expression of pro-inflammatory cytokines and matrix metalloproteinases (MMPs). Importantly, the phenotype could not be transmitted by the transfer of Rsk $2^{y /-}$ bone marrow into hTNFtg mice that demonstrated the essential role for RSK2 expression in mesenchymal cells driving the pathogenesis. In agreement, although no difference in the expression of pro-inflammatory cytokines or MMPs nor a change in apoptosis was detected in synovial fibroblasts isolated from $h T N F$ tg. $R s k 2^{y /-}$, these cells displayed an increased proliferation rate.

Conclusions The anti-proliferative function of RSK2 controls a cell autonomous negative feed-back against the activation of synovial fibroblasts by TNF- $\alpha$, therefore limiting joint destruction in arthritis. Thus, activation of RSK2 is a potential target for the treatment of both local and systemic bone destruction in RA.

\section{A8.15 THE FOCAL CONTACT PROTEIN LASP-1 MODULATES THE MIGRATION CAPACITY OF SYNOVIAL FIBROBLASTS}

doi:10.1136/annrheumdis-2013-203222.15

${ }^{1}$ Adelheid Korb-Pap, 'Denise Beckmann, ${ }^{1} J a n$ Hillen, ${ }^{1,2}$ Marianne Heitzmann, ${ }^{3}$ Catherine S Chew, ${ }^{4}$ Stefan Butz, ${ }^{4}$ Dietmar Vestweber, ${ }^{2}$ Hermann Pavenstädt, ${ }^{1} T h o m a s$ Pap. ${ }^{1}$ Institute of Experimental Musculoskeletal Medicine, University Hospital Muenster, Germany; Internal Medicine D, Department of Nephrology and Rheumatology, University Hospital Muenster, Germany; ${ }^{3}$ Institute of Molecular Medicine and Genetics, Medical College of GA, USA; ${ }^{4}$ Max-Planck-Institute for Molecular Biomedicine, University of Muenster, Germany

Background and Objectives RA synovial fibroblasts (SF) have been suggested to contribute to the spreading of disease through their ability to leave cartilage destruction sites, migrate via the bloodstream and re-initiate the destructive process at distant articular cartilage surfaces. In this context, the actin-crosslinking protein Lasp-1 is of interest, because it is localised at leading edges of migrating cells and regulates metastatic dissemination of different tumours. Therefore, it is particularly important to investigate the role of Lasp-1 in SF migration and its effects on RA.

Materials and Methods To identify different Lasp-1 expression levels in the hind paws of wt and hTNFtg mice, an established model for human RA, Western- blot analyses were performed. In parallel, Lasp-1 expression and its sub-cellular distribution was investigated in SF from wt and hTNFtg mice by Western-blot analyses and immunofluorescence. The migratory capacity of SFs derived from wild-type, Lasp-1\%, hTNFtg and Lasp $1^{-/} /$hTNFtg mice was studied in a modified scratch assay as well as in live cell imaging 